\title{
Editorial
}

\section{Special Issue on Basics and Applications in Quantum Optics}

\author{
Alessia Allevi ${ }^{1,2, *,+}$ (D) Stefano Olivares ${ }^{3,+}$ (D) and Maria Bondani ${ }^{2,+}+(\mathbb{D}$ \\ 1 Department of Science and High Technology, University of Insubria, Via Valleggio 11, 22100 Como, Italy \\ 2 Institute for Photonics and Nanotechnologies, CNR, Via Valleggio 11, 22100 Como, Italy; \\ maria.bondani@uninsubria.it \\ 3 INFN, Milano Section, Department of Physics "Aldo Pontremoli", University of Milan, Via Celoria 16, \\ 20133 Milano, Italy; stefano.olivares@fisica.unimi.it \\ * Correspondence: alessia.allevi@uninsubria.it; Tel.: +39-031-238-6253 \\ + These authors contributed equally to this work.
}

Citation: Allevi, A.; Olivares, S.; Bondani, M. Special Issue on Basics and Applications in Quantum Optics. Appl. Sci. 2021, 11, 10028. https:// doi.org/10.3390/app112110028

Received: 15 October 2021

Accepted: 22 October 2021

Published: 26 October 202

Publisher's Note: MDPI stays neutral with regard to jurisdictional claims in published maps and institutional affiliations.

Copyright: (c) 2021 by the authors. Licensee MDPI, Basel, Switzerland. This article is an open access article distributed under the terms and conditions of the Creative Commons Attribution (CC BY) license (https:// creativecommons.org/licenses/by/ $4.0 /)$.

\section{Introduction}

Quantum technologies are advancing very rapidly and have the potential to innovate communication and computing far beyond current possibilities. Among the possible platforms suitable to run quantum technology protocols, in the last decades quantum optics has received a lot of attention for the handiness and versatility of optical systems. In addition to studying the fundamentals of quantum mechanics, quantum optical states have been exploited for several applications, such as quantum-state engineering, quantum communication and quantum cryptography protocols, enhanced metrology and sensing, quantum optical integrated circuits, quantum imaging, and quantum biological effects. In this Special Issue, we collect some papers and also a review on some recent research activities that show the potential of quantum optics for the advancement of quantum technologies.

\section{Quantum Optics Applications}

The topics addressed in the Special Issue range from quantum computing to quantumstate engineering, from quantum communication to quantum cryptography, from quantum simulation to quantum imaging, in perfect agreement with the four pillars of the European Commission Quantum Technologies Flagship Program.

The first paper [1] of this Special Issue, authored by A. Candeloro et al., focuses on an enhanced version of an all-optical system used to implement a quantum finite automaton [2]. The considered automaton recognizes a well-known family of unary periodic languages that play a crucial role in Descriptional Complexity Theory and in the area of Formal Language Theory. The performance of the device benefits from considering the orthogonal output polarizations of the employed single photons. Moreover, the effect of the detector dark counts on the proper operation of the automaton is taken into account. This kind of photonic quantum automaton could be hardwired into "hybrid" architectures that combine classical and quantum components to build very succinct finite-state devices operating in environments where dimension and energy absorption are particularly critical issues.

The paper written by G. Chesi et al. addresses the topic of quantum-state engineering. The authors present the generation and characterization of Sub-Poissonian states by means of conditional measurements performed on multi-mode twin-beam states [3]. These measurements are based on the use of Silicon photomultipliers [4], a class of photon-number-resolving detectors. Such detectors, very compact and cheap, can open new perspectives in the field of quantum optics and quantum technologies, being suitable for investigating mesoscopic states of light [5]. In the paper, a comprehensive model taking into account all the features of the employed detectors is developed and experimentally verified.

In their paper, M.-S. Kang et al. develop a quantum message authentication protocol for improving security against an existential forgery by means of single-qubit unitary operations [6]. The protocol consists of two parts: a quantum encryption and a correspondence 
check. The first part is realized by means of a linear combination of wave plates [7], while the second one is performed using the Hong-Ou-Mandel interference [8]. The successful experimental implementation of the protocol proves that the employed optical system can be considered as the base technology for a complete quantum cryptosystem providing confidentiality, authentication, integrity, and nonrepudiation.

Furthermore, the paper written by K. Park et al. is devoted to quantum-state engineering [9]. Starting form the recent proposal of obtaining high-purity bi-photon states without degrading brightness and collection efficiency by means of a nonlinear interferometer [10], the authors experimentally investigate the fine tunability of the nonlinear interference method to match constructive interference patterns, while maintaining the high spectral purity of the biphoton state. Their results enrich the usefulness and practicality of the method based on the nonlinear interferometer for the efficient generation of photon pairs with high spectral purity, which represents an excellent practical source for quantum information protocols.

The paper authored by A. Allevi et al. focuses on the role of losses in the degradation of the nonclassicality of mesoscopic quantum states of light to be used for secure data transmission in quantum communication protocols [11]. In particular, the authors investigate, both theoretically and experimentally, the effect caused by two realistic kinds of statisticallydistributed amounts of loss, namely a Gaussian distribution and a log-normal one, on the nonclassical photon-number correlations between the two parties of multi-mode twin-beam states [12]. The achieved results show to what extent the involved parameters, both those connected to loss and those describing the employed states of light, preserve nonclassicality.

In the last research paper, J. Liñares et al. present the physical simulation of the dynamical and topological properties of atom-field quantum interacting systems by means of integrated quantum photonic devices [13]. The photonic device consists of integrated optical waveguides supporting two collinear modes, which are coupled by integrated optical gratings [14]. The two-mode photonic device with a single-photon quantum state represents the quantum system, and the optical grating corresponds to an external field. This photonic simulator can be regarded as a basic brick for constructing more complex photonic simulators.

Finally, in the review paper by C. Abbattista et al. the advancement of the research toward the design and implementation of quantum plenoptic cameras is presented and discussed [15]. At variance with standard plenoptic cameras, these devices have dramaticallyenhanced features, such as diffraction-limited resolution, large depth of focus, and ultra-low noise [16]. For the quantum advantages of the proposed devices to be effective and appealing to end-users, the authors propose to develop high-resolution single-photon avalanche photodiode arrays and high-performance low-level programming of ultra-fast electronics, combined with compressive sensing and quantum tomography algorithms, with the aim of reducing both the acquisition and the elaboration time by two orders of magnitude. These new strategies will open the way to new opportunities and applications, such as for biomedical imaging, security, space imaging, and industrial inspection.

Author Contributions: Conceptualization, A.A., S.O. and M.B.; methodology, A.A.; writingoriginal draft preparation, A.A., S.O. and M.B; writing-review and editing, A.A., S.O. and M.B. All authors have read and agreed to the published version of the manuscript.

Funding: This research received no external funding.

Institutional Review Board Statement: Not applicable.

Informed Consent Statement: Not applicable.

Data Availability Statement: The datasets used and analysed during the current study are available from the corresponding author on reasonable request. 
Acknowledgments: This issue would not have been possible without the contributions of several valued authors, professional reviewers, and the Applied Sciences editorial team. We first extend our congratulations to all the authors. Second, we would like to take this opportunity to show our sincere gratitude to all reviewers. Finally, we thank the editorial team of Applied Sciences and especially Patrick Han.

Conflicts of Interest: The authors declare no conflict of interest.

\section{References}

1. Candeloro, A.; Mereghetti, C.; Palano, B.; Cialdi, S.; Paris, M.G.A.; Olivares, S. An enhanced photonic quantum finite automaton. Appl. Sci. 2021, 11, 8768. [CrossRef]

2. Mereghetti, C.; Palano, B.; Cialdi, S.; Vento, V.; Paris, M.G.A.; Olivares, S. Photonic realization of a quantum finite automaton. Phys. Rev. Res. 2020, 2, 013089. [CrossRef]

3. Chesi, G.; Allevi, A.; Bondani, M. Conditional Measurements with Silicon Photomultipliers. Appl. Sci. 2021, 11, 4579. [CrossRef]

4. Akindinov, A.V.; Martemianov, A.N.; Polozov, P.A.; Golovin, V.M.; Grigoriev, E.A. New results on MRS APDs. Nucl. Instrum. Methods Phys. Res. Sect. A 1997, 387, 231-234. [CrossRef]

5. Cassina, S.; Allevi, A.; Mascagna, V.; Prest, M.; Vallazza, E.; Bondani, M. Exploiting the wide dynamic range of silicon photomultipliers for quantum optics applications. EPJ Quantum Technol. 2021, 8, 4. [CrossRef]

6. Kang, M.-S.; Kim, Y.-S.; Choi, J.-W.; Yang, H.-J.; Han, S.-W. Experimental Quantum Message Authentication with Single Qubit Unitary Operation. Appl. Sci. 2021, 11, 2653. [CrossRef]

7. Clarke, R.B.M.; Kendon, V.M.; Chefles, A.; Barnett, S.M.; Riis, E.; Sasaki, M. Experimental realization of optimal detection strategies for overcomplete states. Phys. Rev. A 2001, 64, 012303. [CrossRef]

8. Horn, R.T.; Babichev, S.; Marzlin, K.-P.; Lvovsky, A.; Sanders, B.C. Single-qubit optical quantum fingerprinting. Phys. Rev. Lett. 2005, 95, 150502. [CrossRef] [PubMed]

9. Park, K.; Lee, D.; Shin, H. Tunability of the Nonlinear Interferometer Method for Anchoring Constructive Interference Patterns on the ITU-T Grid. Appl. Sci. 2021, 11, 1429. [CrossRef]

10. Cui, L.; Su, J.; Li, J.; Liu, Y.; Li, X.; Ou, Z.Y. Quantum state engineering by nonlinear quantum interference. Phys. Rev. A 2020, 102, 033718. [CrossRef]

11. Allevi, A.; Bondani, M. Tailoring Asymmetric Lossy Channels to Test the Robustness of Mesoscopic Quantum States of Light. Appl. Sci. 2020, 10, 9094. [CrossRef]

12. Allevi, A.; Bondani, M. Preserving nonclassical correlations in strongly unbalanced conditions. J. Opt. Soc. Am. B 2019, 36, 3275-3281. [CrossRef]

13. Liñares, J.; Prieto-Blanco, X.; Carral, G.M.; Nistal, M.C. Quantum Photonic Simulation of Spin-Magnetic Field Coupling and Atom-Optical Field Interaction. Appl. Sci. 2020, 10, 8850. [CrossRef]

14. Lee, D. Electromagnetic Principles of Integrated Optics; Wiley: New York, NY, USA, 1986.

15. Abbattista, C.; Amoruso, L.; Burri, S.; Charbon, E.; Di Lena, F.; Garuccio, A.; Giannella, D.; Hradil, Z.; Iacobellis, M.; Massaro, G.; et al. Towards Quantum 3D Imaging Devices. Appl. Sci. 2021, 11, 6414. [CrossRef]

16. Pepe, F.V.; Di Lena, F.; Mazzilli, A.; Garuccio, A.; Scarcelli, G.; D’Angelo, M. Diffraction-limited plenoptic imaging with correlated light. Phys. Rev. Lett. 2017, 119, 243602. [CrossRef] [PubMed] 